\title{
ACTITUDES LINGÜÍSTICAS HACIA EL MAPUDUNGUN Y EL CASTELLANO: ESTUDIO EXPLORATORIO EN DOS COMUNIDADES PEWENCHE DEL ALTO BIOBÍO*1
}

\section{LANGUAGE ATTITUDES TOWARDS MAPUDUNGUN AND SPANISH: AN EXPLORATORY STUDY IN TWO PEWENCHE COMMUNITIES FROM ALTO BIOBÍO}

\author{
Marisol Henríquez Barahona** \\ Universidad Católica de la Santísima Concepción, Chile \\ mhenriquez@ucsc.cl \\ Javiera Dinamarca Zurita*** \\ Universidad Católica de la Santísima Concepción, Chile \\ jdinamarca@ucsc.cl
}

\section{Resumen:}

En el presente artículo se examinan y describen las principales actitudes lingüísticas que los hablantes bilingües de dos comunidades pewenche de la comuna de Alto Biobío manifiestan hacia el mapudungun y el castellano. Este estudio se inscribe en el ámbito de la sociolingüística, es de carácter exploratorio y tiene un enfoque cualitativo. Los datos analizados corresponden al discurso de 20 hablantes bilingües, pertenecientes a las comunidades de Pitril y Butalelbun. En términos generales, los resultados muestran que los hablantes tienen actitudes sociolingüísticas positivas hacia el mapudungun, que se justifican en el vínculo inextricable que existe entre la lengua y la identidad étnica; no obstante, en Pitril, se aprecia una tensión entre el subsistema afectivo y conativo de la actitud. Por último, los hablantes tienen una visión utilitaria del castellano que podría incidir en la vitalidad del mapudungun.

Palabras claves: actitudes lingüisticas, bilingüismo, castellano, mapudungun, pewenche

\section{Abstract:}

This paper describes a group of pewenche bilingual speakers' language attitudes towards Mapudungun and Spanish. This study is part of the field of sociolinguistics, is exploratory in nature and has a qualitative approach. The analyzed data correspond to the speech of 20 bilingual speakers, belonging to the ethnic communities of Pitril and Butalelbun.

In general, findings show that speakers have positive sociolinguistic attitudes towards Mapudungun, which are justified in the inextricable bond between language and ethnic identity; however, in Pitril, there is a tension between the affective and conative subsystem of attitude. Finally, the speakers have a utilitarian view of Spanish that could affect the ethnic language's vitality.

Key words: language attitudes, bilingualism, Spanish, mapudungun, pewenche

Recibido: 25 de mayo de 2018

Aceptado: 06 de noviembre de 2018

\footnotetext{
*Los resultados de este artículo están asociados al proyecto Fondecyt Iniciación 11150508: “Contacto mapudungun castellano: rasgos fonético-fonológicos del castellano presentes en el chedungun de escolares pewenches del Alto BíoBío", que dirige la primera autora.

** Chilena. Doctora en Lingüística de la Universidad Católica de la Santísima Concepción, Concepción, Chile. mhenriquez@ucsc.cl

*** Chilena. Magíster en Linguiística Aplicada de la Universidad Católica de la Santísima Concepción, Concepción, Chile.jdinamarca@ucsc.cl
} 


\section{Introducción}

El estudio de las actitudes lingüísticas ha sostenido en las últimas décadas un interés creciente dentro de la investigación sociolingüística, por cuanto se ha comprobado que lo que el hablante siente y piensa con respecto a su lengua influye significativamente en ésta y en sus prácticas sociolingüísticas (Moreno Fernández, 2009; Silva-Corvalán y Enrique Arias, 2017). Los aportes de su estudio se proyectan en diversos ámbitos, pues no solo permiten comprender las creencias y las posturas valorativas que subyacen al uso lingüístico y a los diversos fenómenos relacionados con éste; sino que proveen información sustancial sobre los hablantes, su posición dentro de un grupo social, los prejuicios lingüísticos y la salud de las lenguas minoritarias (Blas Arroyo, 2005; Moreno Fernández, 2009; Serrano, 2011; Díaz Campos, 2014; Silva- Corvalán y Enrique-Arias, 2017). En este último sentido, según Garrett (2010), las actitudes lingüísticas desempeñan un papel clave en la supervivencia de lenguas minoritarias, porque inciden en las decisiones de los padres de continuar o no con la transmisión y uso de éstas, afectando de este modo su proyección.

En Chile, los estudios de actitudes sociolingüísticas hacia el mapudungun se han direccionado a observar las reacciones subjetivas de los hablantes hacia su lengua o las lenguas en contacto (Croese, 1983; Fernández y Hernández, 1984; Lagos, 2006; 2017; Gundermann, Canihuán, Castillo, Clavería, 2008; Olate y Henríquez, 2010; Gundermann, 2014; entre otros). Los aportes de estos trabajos han sido valiosos para comprender la dinámica de funcionamiento de las lenguas y la complejidad de las valoraciones sociales que subyacen a los usos y elecciones linguiísticas. No obstante, el desarrollo indagativo en esta área continúa siendo una necesidad, debido a la compleja situación de vulnerabilidad que actualmente enfrenta la lengua ancestral, producto de las consecuencias del contacto histórico y la dominancia social, política y cultural que afecta al pueblo mapuche.

En este contexto se hace necesario examinar las posturas valorativas de los hablantes mapuche y avanzar en la comprensión de las dinámicas de funcionamiento 
social del chedungun ${ }^{2}$ en contacto con el castellano; asimismo, pretendemos proveer datos relacionados al proceso de cambio que vive la lengua originaria y su proyección en este territorio específico.

\subsection{Algunos Conceptos Teóricos}

\subsection{Actitud Lingüística}

El concepto de actitud es probablemente uno de los más controvertidos y complejos de delimitar en el ámbito de las ciencias sociales, especialmente en el campo de la psicología social, donde ha tenido una larga y rica trayectoria. De manera general, se define como la disposición a evaluar, favorable o desfavorablemente, una entidad, una idea, un lugar o un objeto particular, por lo que su naturaleza valorativa es uno de los componentes definitorios (Albarracín, Zanna, Johnson y Kumkale, 2005; Edward, 2009).

En el campo de la sociolingüística, la actitud se entiende como la postura crítica de un hablante hacia fenómenos específicos de una lengua o, incluso, hacia variedades lingüísticas o rasgos de ésta (Blas Arroyo, 2005; Serrano, 2011). Para Moreno Fernández (2009), se trata de una manifestación de la actitud social de los individuos, distinguida por centrarse y referirse específicamente tanto a la lengua como al uso que de ella se hace en la sociedad. Es un reflejo de las actitudes psicosociales, de hecho, según este autor, es una actitud psicosocial.

La actitud lingüística deriva de la actitud social, pero se diferencia de ésta, porque se enfoca en los fenómenos lingüísticos que percibe el hablante y en la conducta comunicativa de los interlocutores. Así, estas reacciones o valoraciones se pueden manifestar hacia determinados usos discursivos, hacia lenguas hegemónicas y minoritarias y hacia múltiples fenómenos sociolingüísticos. Se caracterizan por ser dicotómicas, positivas o negativas ${ }^{3}$; individuales o colectivas. Además, son dinámicas, puesto que cambian constantemente por la influencia de diversos factores, como el género, la edad, el contexto lingüístico, entre otros. Finalmente, se caracterizan por estar

\footnotetext{
${ }^{2}$ Variante pewenche del mapudungun o lengua mapuche. Los colaboradores que participaron de este estudio, denominan chedungun y/o mapudungun a su lengua.

${ }^{3}$ Para algunos especialistas, las actitudes no pueden ser neutras, si esto ocurriera se debería hablar de ausencia de actitud (López Morales, 2004).
} 
influenciadas por la experiencia social del hablante y su comunidad (Moreno Fernández, 2009).

\subsection{Componentes de la actitud lingüística}

Entre los especialistas, el modelo de actitudes que suscita mayor consenso para ofrecer una explicación de este fenómeno es el de tres componentes: 1) un subsistema afectivo, que involucra las relaciones emocionales y los sentimientos que surgen en la interacción y que están dirigidos a la lengua; 2) un subsistema cognitivo, que involucra todos los prejuicios y creencias que tienen los hablantes sobre la lengua y sobre el proceso comunicativo y finalmente, 3) un subsistema conductual, que refleja el comportamiento o la predisposición a actuar de determinadas formas, respecto del objeto actitudinal (Hernández Campoy, 2004; Garret, 2010; Díaz Campos, 2014). De éstos, el subsistema afectivo es sumamente relevante, puesto que actúa como un barómetro que incide en la intensidad evaluativa. Puede influir en la aceptación y en el rechazo del objeto evaluado (Garrett, 2010).

Ahora bien, aunque existe unanimidad con respecto a los elementos que componen la actitud lingüística, es posible observar cierta dificultad a la hora de determinar la congruencia entre estos tres subsistemas, ${ }^{4}$ pues en algunas ocasiones parecen no estar alineados coherentemente. Según Garrett (2010), la controversia se centra en el tercer componente del modelo -el conductual- ya que hay instancias en las que entra en conflicto y tensión con los otros, lo que podría generar una asimetría o desarticulación. Así, por ejemplo, en una comunidad los hablantes podrían tener valoraciones positivas hacia una lengua, pero evitar hacer uso de ella por diversos factores, como las creencias, los prejuicios, el prestigio lingüístico, etc. Para el caso de la comunidad de habla mapuche, esto tiene relación con lo que Croese denominaba conflicto de intereses (1983).

\subsection{Método}

La presente investigación, de naturaleza exploratoria, se adscribe al paradigma cualitativo y corresponde a un estudio de caso. El objetivo principal que nos hemos propuesto es describir las principales actitudes lingüísticas hacia el chedungun y el castellano en hablantes bilingües de dos comunidades pewenche del Alto Biobío.

3.1 Los participantes y la recogida de datos

\footnotetext{
${ }^{4}$ Este es un tema importante y controversial en el campo de las actitudes lingüísticas.
} 
Para el logro del objetivo planteado y de acuerdo con el diseño del estudio, trabajamos con una muestra selectiva e intencionada compuesta por un grupo de 20 hablantes pertenecientes a las comunidades de Butalelbun y Pitril del Valle del Queuco (10 colaboradores de cada comunidad). Los participantes tienen entre 18 y 75 años y son hablantes bilingües de chedungun.

Para la recogida de los datos diseñamos y aplicamos una entrevista en profundidad, que incorporaba diversos tópicos relacionados con el uso y la valoración social hacia ambas lenguas. Luego de obtener los permisos de las autoridades tradicionales de cada comunidad, entrevistamos de manera individual a nuestros colaboradores durante un tiempo aproximado de 60 minutos. Este material, se complementó con las observaciones y con los registros in situ realizados por las investigadoras en el trabajo de campo desarrollado en la zona.

Todos los entrevistados participaron de manera voluntaria y nos dieron su consentimiento oral informado según protocolo del Proyecto Fondecyt 11150508 al cual se adscribe este estudio.

Con respecto a la organización y al procesamiento de los datos, es necesario mencionar que se utilizó el software Nvivo 11 que facilitó la codificación, almacenamiento y análisis de los datos entregados por los hablantes.

\subsection{Resultados}

Los hallazgos de esta investigación se organizarán y describirán en torno a tres dimensiones: en la primera se examinan las actitudes de orgullo y fidelidad que manifiestan los hablantes hacia el chedungun; en la segunda, se describe la tensión o conflicto actitudinal y, finalmente, se aborda la actitud utilitaria hacia el castellano.

\subsection{Actitudes lingüísticas hacia el chedungun}

Según lo observado, los miembros de ambas comunidades presentan valoraciones altamente positivas hacia el chedungun que se asocian con los valores tradicionales, la identidad étnica, la vida familiar y comunitaria. Para estos hablantes, su lengua se consolida como el medio principal para las interacciones lingüísticas de intimidad, confianza y solidaridad (Henríquez, 2014). Se considera una lengua de relaciones internas, que genera sentimientos positivos de afecto y orgullo étnico (Gundermann, 2014 y Henríquez, 2015). Sin embargo, aunque los participantes de Pitril y Butalelbun 
manifiestan actitudes positivas, se observan algunas diferencias entre ambas comunidades.

\subsubsection{Fidelidad y orgullo}

En este estudio, la fidelidad lingüística se entiende como el deseo que tiene la comunidad de preservar sus prácticas lingüísticas originarias y de defender su lengua de intervenciones y de elementos linguiísticos externos (Blas Arroyo, 2005); el orgullo en tanto, se relaciona con el grado de identificación que los hablantes tienen con su lengua como un elemento fundamental para la identidad cultural. Además, se manifiesta en el entusiasmo y satisfacción que sienten los participantes por hablar y/o aprender chedungun. Según Brezinger (1997), esta última actitud es la más importante para el mantenimiento de una lengua minoritaria.

El orgullo y la fidelidad hacia el chedungun se observan cuando los participantes de ambas comunidades señalan que su lengua representa un bien comunitario y un símbolo de identidad territorial y cultural que les permite diferenciarse de los demás:

Yo me represento a través de mi lengua en cualquier parte que ando y me representa que soy mapuche, $[\ldots]$, porque al hablar castellano uno no se diferencia de nadie. (M, V., Butalelbun).

Para mí, hablar mapudungun significa posicionarme como mapuche, posicionarme como [parte de] una nación con una identidad propia, para mí eso es lo primordial y dentro del contexto actual en que nosotros hoy día estamos, para nosotros es una necesidad para poder posicionarnos a partir del chedungun, para mí es uno de los elementos más clave y eso es lo que significa acá [...] en el actualmente llamado territorio Chile (G, P., Butalelbun).

Estas actitudes también se aprecian cuando los colaboradores declaran sentirse honrados y felices de hablar su lengua patrimonial:

Me siento bien [cuando hablo chedungun], es algo que es mío y es algo que me gusta, algo que me nace, algo [con lo] que crecí, entonces yo no podría cambiar a otra lengua por la que tengo $(F, P$., Butalelbun).

Mira, cuando me toca hablar en chedungun... eh no sé, en una asamblea, practicar el chedungun... para mí es un orgullo, un orgullo porque me identifico, o sea no tengo porqué avergonzarme de mi lengua, soy pewenche y orgulloso de ser pewenche... y hablar [...] es un orgullo, me siento feliz porque, soy escuchado, alguien me está escuchando (L, LL., Pitril) 
Sí, por ejemplo, cuando nosotros nos vemos con personas que saben hablar o crecimos juntos, nos comunicamos, nos entendemos bien, conocemos todo, como crecimos, estamos más cómodos, nos sentimos más cómodos [hablando chedungun] (F, P., Butalelbun).

Estos discursos no sólo muestran los sentimientos de orgullo, comodidad e identificación de los hablantes con su idioma, sino también la fidelidad, especialmente cuando declaran sentirse reticentes a postergar y permutar el chedungun por el castellano o aceptar intervenciones o elementos foráneos en su lengua. En este sentido, la actitud de fidelidad se relaciona con la ideología del purismo lingüístico que se observa en ambas comunidades, especialmente en los hablantes mayores, quienes recalcan la importancia de preservar una variedad del chedungun que no tenga influencias de la lengua oficial:

[El chedungun] está más cambiado, no sé pues, la juventud pues, porque los viejitos ya fallecieron y entonces como sigue la gente joven... entonces ellos cambian el chedungun... hablan su idioma y mezclan [...] eso no, pues, no está bien, porque ellos tienen que seguir la conversa de antes que tenían sus abuelos, abuelitas. (R, G., Pitril)

En Pitril, la fidelidad se puede apreciar en la mantención de la lengua patrimonial dentro de los contextos intracomunitarios, íntimos (hogares) y ceremoniales. No obstante lo anterior, el uso del chedungun no es exclusivo y, según los colaboradores, ha disminuido considerablemente, sobre todo entre los más jóvenes y niños, porque la lengua oficial ha permeado los espacios comunicativos que tradicionalmente le pertenecían al mapudungun ${ }^{5}$ (Henríquez, 2015). Sin embargo, esto no ha afectado las valoraciones positivas de los hablantes.

En Butalelbun el panorama es distinto, la fidelidad se observa en la constante transmisión y uso del idioma en todos los espacios sociales comunitarios tradicionales y no tradicionales. Para estos hablantes, el chedungun constituye un elemento esencial para la configuración de la identidad étnica, por tanto su enseñanza y uso es asumido como un deber. Así, quienes dominan, hablan y enseñan el chedungun son valorados y

${ }^{5}$ Esto evidencia el proceso de desplazamiento de la lengua patrimonial en Pitril, lo que concuerda con los hallazgos realizados en el ámbito fonológico en la zona (Henríquez y Salamanca, 2015). 
reconocidos por sus familiares, vecinos y amigos, porque tienen acceso al conocimiento o kimün pewenche. Por lo mismo, cumplen un rol fundamental en la preservación de prácticas lingüísticas y culturales ancestrales que se relacionan con el mantenimiento de la espiritualidad pewenche. El prestigio y estatus que se les concede en la zona se vincula, además, con la lealtad o resistencia lingüística que han demostrado, al persistir con el uso de la lengua, pese a todas las instancias de violencia, racismo y discriminación sufridas.

Por el contrario, no hablar u olvidar la lengua es mal evaluado, porque según los hablantes evidenciaría el awingkamiento ${ }^{6}$ del pewenche, como lo expresa este colaborador:

no es pewenche porque no habla en chedungun, porque quieren andar mintiendo y no quiere hablar y uno no puede olvidarse de su lengua que tiene, de ninguna manera uno puede olvidar, aunque de chico que viva afuera, siempre anden junto con los colonos, uno no se puede olvidar, [...] yo tengo una cuñada que vive en Santiago y que ella no, que ya no podía hablar, que se le olvidó porque hace mucho tiempo que está trabajando allá; pero ella dice que se le olvidó ya hablar, pero no... no es verdad. Porque ella no quiere hablar no más el chedungun. [...] porque les da vergüenza, ahí no sé poh. [...] eso es, les da vergüenza ser pewenche y ellos se creen, que son criollos, así... se creen wingkas (H. M., Butalelbun)

Este tipo de actitudes puede generar incluso discriminación entre pares, como lo manifiesta un participante de Pitril, quien no posee una competencia productiva avanzada en la lengua y señala haber sido discriminado por este motivo:

[...] cuando yo estudié, igual, fui súper discriminado... por ambas partes [colonos y pewenche] como yo me fui a Ralko allá siempre hubo más personas no pewenche, la mayoría, y los que eran pewenche eran súper pocos, contados con la mano... y la mayoría era de Butalelbun y de Trapa Trapa [...] los de Trapa Trapa, sí eran hablantes netamente y ellos me discriminaban porque yo no sabía hablar (A, Ll., Pitril).

Esto también es señalado por una colaboradora de Butalebun, quien admite haber sido víctima de discriminación por los miembros de su propia comunidad y familia:

\footnotetext{
${ }^{6}$ El awingkamiento hace referencia a un proceso de chilenización u occidentalización que evidencian algunos mapuche/pewenche según la opinión de sus pares. Este proceso es valorado negativamente por los miembros de esta comunidad.
} 
En mi comunidad es muy mal visto la persona que habla puro wingkadungun, sobre todo en mi familia, porque como fui discriminada yo [por no hablar el chedungun] así se discrimina allá por no hablar... se cree que los que no hablan se creen wingkas ( $\mathrm{J}$, M., Butalelbun).

Lo señalado puede analizarse desde dos perspectivas: por un lado, estas reacciones negativas generan ciertas tensiones en las relaciones internas del grupo; pero por otro, refuerzan las valoraciones positivas hacia el uso y mantenimiento de la lengua.

\subsection{Conflicto actitudinal}

Como se ha señalado, en ambas comunidades el chedungun se considera un símbolo y un factor determinante para la mantención de la identidad étnica y de las dinámicas culturales. Se asocia con los valores y costumbres tradicionales, por lo tanto, su mantención y transmisión genera sentimientos y valoraciones muy positivas. No obstante, en Pitril, pese a estas valoraciones, se observa un uso muy limitado de la lengua y un evidente proceso de interrupción de la transmisión. Esta tensión, que Cróese (1983) denomina “conflicto de interés" y Gundermann (2014) “ambivalencia”, se refiere al conflicto entre el deseo de preservar y desarrollar el uso del chedungun y la necesidad de asumir el castellano como vehículo principal de comunicación. Este conflicto es el resultado de la tensión entre el sistema afectivo y el conativo en la actitud linguística del sujeto (Garrett, 2010) y es propio de comunidades en situación de contacto y minorización lingüística.

En el caso de este estudio, una de las causas de esta tensión actitudinal se explicaría por la creencia que tienen algunos padres y abuelos de Pitril de que el bilingüismo chedungun-castellano es un obstáculo para el progreso estudiantil y para el aprendizaje de la lengua oficial, de modo que deciden dejar de enseñar chedungun para que los niños se desarrollen académicamente:

Fíjate yo, para decirla sinceramente que a los hijos míos los primeros años, cuando tenían dos años, tres años, no quisimos hablar en chedungun, entonces para que también salieran a estudiar (R, G., Pitril).

En esta misma línea, la visión utilitaria que los hablantes tienen del castellano, refuerza este tipo de actitudes; porque lo conciben como un idioma que permite la 
comunicación intercultural y que propicia la movilidad socioeconómica, lo cual se contrapone con la representación del chedungun como una lengua de relaciones internas que remite a la identidad territorial, religiosa y cultural (Ojeda y Álvarez, 2014; Dinamarca, 2017). La tensión entre lo discursivo y lo pragmático se refuerza con la presión que ejerce la sociedad dominante a través de sus políticas lingüísticas monoglósicas que no ceden espacio a las lenguas minoritarias.

Sumado a lo anterior, y de acuerdo con lo observado durante el trabajo de campo, los habitantes adultos de Pitril perciben que el chedungun, aunque es un símbolo de identidad y orgullo, también es un estigma que suscita la discriminación y la violencia simbólica de parte de la sociedad chilena; en consecuencia, algunos deciden no transmitirlo para evitarles a sus hijos y nietos la experiencia traumática que ellos vivieron durante su niñez y su trayectoria escolar:

Entonces si hablaban en chedungun los mismos profesores se reían de los niños que hablaban en chedungun, entonces se fueron dando cuenta que los profesores se estaban burlando de lo que ellos estaban hablando, entonces se fue perdiendo un poco el chedungun [en las familias] ya no lo querían enseñar (G, B., Pitril).

No obstante, en esta misma comunidad existe una consciencia y preocupación con respecto a la vitalidad de la lengua y su futuro. Esta se observa principalmente entre los jóvenes que cuestionan la ambivalencia de los mayores y la decisión que han tomado algunos de no transmitir la lengua.

[...] es que por eso le digo, toda la gente acá que es pewenche, que participa en el ngillatun, que conoce el tema... predica pero no practica... o sea gran parte no todos, estoy exagerando, gran parte... no solamente de esta comunidad, de Cauñicu igual... señoras, señoras abuelitas que dicen "no si hay que hablar el chedungun" "tienen que enseñarle a hablar a sus niños, a sus hijos porque tenemos que recuperar nuestra cultura, no perderlo" pero resulta que el nieto de ella que vive con ella no sabe nada de chedungun...entonces ien qué estamos? (A., Ll., Pitril).

Estos mismos jóvenes, conscientes de la importancia de la revitalización de la lengua, forman parte de una agrupación comunitaria que lleva a cabo procesos de inmersión y de enseñanza de la lengua dirigidos a jóvenes y niños del territorio que han olvidado o no saben chedungun. Estos procesos autogestionados se denominan 
internados lingüísticos y vienen desarrollándose en la zona desde hace algunos años. Según diversos especialistas, este tipo de acciones, que emergen desde las propias comunidades, son fundamentales para la recuperación de lenguas amenazadas, ya que son los propios hablantes quienes deben plantear la necesidad de re-utilizarlas y reaprenderlas (Fishman, 1989; Enrique López, 2015).

Por último, este conflicto no se observó en los discursos de los colaboradores de Butalelbun, donde la lengua no sólo es valorada como símbolo identitario sino que es el vehículo de comunicación principal, por lo que su transmisión es constante y sistemática.

\subsection{La utilidad del castellano}

Como se mencionó anteriormente, los hablantes de ambas comunidades manifiestan una actitud utilitaria hacia el castellano. Es decir, todos los colaboradores reconocen que la lengua oficial es una herramienta que les permite comunicarse y conectarse con la sociedad chilena, que asegura oportunidades económicas y de movilidad social a través del trabajo y la educación:

También el castellano igual es muy importante porque uno no tiene que aprender el puro chedungun, porque si yo converso así, voy a Ralko, converso con alguien, a mí no me van a entender lo que yo digo (R, G., Pitril).

Esta visión utilitaria se encuentra fuertemente influenciada por la ideología del mercado y el capital lingüístico (Bourdieu, 1985), que sugiere que el perfil económico, político y social de una sociedad se encuentra ligado inherentemente a su perfil lingüístico, por tanto, para poder desenvolverse competentemente dentro de ésta, es necesario que los hablantes de la comunidad minoritaria adquieran, utilicen y prioricen la lengua mayoritaria:

[...] Como le decía, el castellano es para comunicarme con todo lo que es, con todo Chile por decirle, porque ahí nos entendemos. Es la lengua que nos permite comunicarnos afuera. [...] para mí es hablar con las empresas, con las instituciones, me entiendo, puedo conseguir recursos, claro, entonces no es malo... (L, Ll., Pitril). 
Ahora bien, aun cuando los participantes de este estudio enfatizan en la utilidad que tiene la lengua oficial, también señalan que ésta simboliza un mecanismo de sometimiento, que no les agrada, porque representa la imposición de una tradición idiomática, cultural, socioeconómica y política de la sociedad hegemónica (Durán y Ramos, 1986, 1989; Olate, Alonqueo y Caniguan, 2013; Olate, 2017). En este sentido, ellos son conscientes de que si no hablan castellano, no serán reconocidos ni validados dentro de la sociedad dominante. Así, los pewenche se han visto forzados a adquirir y priorizar la lengua oficial para sobrevivir en la sociedad dominante:

El castellano es un sometimiento que tenemos nosotros como indígenas, porque nosotros para ser competentes en la sociedad tenemos que aprender hablar el [castellano] yo creo que nosotros como mapuche usamos la interculturalidad... no así el wingka con nosotros. Nosotros tenemos que aprender a hablar el wingkadungun para no ser discriminados, para poder ser competentes en la sociedad, para poder tener estudios, para poder defendernos, para saber cómo funciona todo el sistema... el wingkadungun es una herramienta que yo tengo para poder ser competente, pero más allá... si me identifica o si me gusta... claramente no me gusta y me cuesta... (J, M., Butalelbun)

En este contexto, la imposición del castellano se presenta como una consecuencia del proceso de colonización (Olate, 2017), y claramente, esta lengua no tiene el mismo valor cultural y emocional que sí posee el chedungun.

Con respecto a la influencia de esta actitud en el comportamiento sociolingüístico del hablante bilingüe, se puede señalar que la visión utilitaria de la lengua oficial podría afectar la vitalidad del chedungun. Sin embargo, a pesar de las presiones globalizantes del mundo moderno, los hablantes de ambas comunidades siguen haciendo uso de su idioma, lo que se traduce en un acto de resistencia frente a la asimilación cultural y a las condicionantes que impone la sociedad hegemónica.

\subsection{Reflexiones finales}

En términos generales, en ambas comunidades se evidencian valoraciones altamente positivas hacia el chedungun. En los discursos de los hablantes se expresan sentimientos de orgullo y fidelidad que se sustentan en la concepción de la lengua como un bien comunitario que representa los valores tradicionales, la vida familiar y que propicia la comunicación entre pares. Estas valoraciones se corresponden con la representación de 
la lengua como un símbolo identitario esencial, que distingue a sus usuarios de los miembros de la sociedad dominante.

La fidelidad hacia la lengua, por otra parte, se visualiza en el deseo de los hablantes por preservar una variedad "pura" del chedungun que no tenga influencias del castellano. En este sentido, la necesidad de conservar y transmitir prácticas lingüísticas ancestrales, que se encuentran amenazadas por el contacto, evidencia esta actitud. En el caso de Butalelbun, ésta también se aprecia en el uso y transmisión sistemática de la lengua y en la alta valoración social hacia quienes la dominan y la usan. Lo contrario, es asumido como un proceso de awingkamiento o pérdida de identidad. En relación con este punto, es necesario agregar la evidente influencia de las ideologías lingüísticas dominantes en las actitudes de los hablantes. Esto se observa en el caso del purismo linguiístico de la comunidad que permea la actitud de fidelidad y que podría ser interpretado como una estrategia comunitaria de defensa lingüística y cultural; pero al mismo tiempo, podría generar la minusvaloración de comportamientos linguiísticos sincréticos y variables; típicos de situaciones de contacto y usualmente presentes en los discursos de jóvenes y niños. Con respecto a esto, creemos importante seguir indagando en estas valoraciones y sus efectos; puesto que la aprobación o desautorización, dependiendo de la cercanía o no a la variante más "pura" o "antigua" atenta contra la variabilidad y, por tanto, podría, perjudicar, sin pretenderlo, el uso de la lengua minorizada, provocando un conflicto y tensión generacional.

Otro aspecto a destacar es el hecho de que en Pitril se observa una tensión entre las actitudes positivas y el mantenimiento del mapudungun. Esta comunidad, por su ubicación geográfica, ha estado expuesta a sistemáticos procesos de intervención, lo que ha impactado en el comportamiento sociolingüístico de sus hablantes. Creemos que la ambivalencia presente es producto de las diversas ideologías y valoraciones sociales que subyacen a las lenguas. El mapudungun, aunque es altamente valorado como símbolo identitario y diferenciador étnico, es a la vez un marcador de bajo nivel educacional, de bajo prestigio y de ausencia de progreso o retraso económico. El español, en cambio, ofrece al hablante la posibilidad de elevar su estatus. Es visto como una herramienta que responde a la necesidad o motivación instrumental que contribuye a la integración y supervivencia. 
Por último, se debe reflexionar sobre el hecho de que las lenguas son, antes que todo, construcciones sociales, culturales e históricamente determinadas (Kroskrity y Field, 2009) por lo que se hace necesario indagar en los sistemas de creencias y valoraciones que poseen sus hablantes. Esto permitirá vislumbrar los efectos que tienen las transformaciones socioculturales e históricas en las prácticas comunicativas y, asimismo, discernir sobre la dinámica de funcionamiento y vitalidad de dichas lenguas; en especial de los procesos de cambio que vive la lengua originaria y su proyección sociolingüística.

\section{Bibliografía}

Albarracín, Dolores, Zanna, M., Johnson, B. y Kumkale, T. The Handbook of Attitudes. Londres: Lawrence Erlbaum, 2005.

Bengoa, José. Historia del pueblo mapuche. Siglos XIX y XX. Santiago;chile: LOM, 2000. Blas Arroyo, José. Sociolingüística del español. Desarrollos y perspectivas en el estudio de la lengua española en contexto social. Madrid: Cátedra, 2005.

Bourdieu, Pierre. ¿Qué significa hablar? La economía de los intercambios lingüísticos. Madrid: AKAL, 1985.

Brezinger, Matthias. "Language Contact and Language Displacement." The Handbook of Sociolinguistics. Ed. Florian Coulmas. Blackwell: Maiden, 1997. 273-284.

Croese, Robert. "Algunos resultados de un trabajo de campo sobre las actitudes de los mapuches frente a su lengua materna". Revista de Lingüística Teórica y Aplicada 21 (1983): 23-34.

Díaz campos, M. Introducción a la sociolingüística hispánica. Singapur:J.Wiley, S., 2014.

Dinamarca, Javiera. "Aproximación a las ideologías y actitudes lingüísticas de hablantes pewenche del Alto Biobío". Tesis. Universidad Católica de la Santísima Concepción, Chile, 2017.

Durán, Teresa y Ramos, N. "Incorporación del español por los mapuches del centro-sur de Chile durante los siglos XVI, XVII y XVIII”. Lenguas Modernas 13(1986): 17-36.

Durán, Teresa y Ramos, Nelly. "Interacción mapudungun-castellano vinculada a contextos educacionales en una población rural." Lenguas Modernas 16(1989): 97-114.

Edward, John. Language and Identity. An introduction. Cambridge: Cambridge University Press, 2009.

Enríquez López, Luis. "El hogar, la comunidad y la escuela en la revitalización de lenguas originarias de América Latina" Eds. Luis Enrique López, Ruth Moya y Matthias Abram. Pueblos indígenas y educación No. 64. Quito: Abya-Yala, 2015. 211-338.

Fasold, Ralph. La sociolingüística de la sociedad. Introducción a la sociolingüística. Madrid: Visor Libros, 1996.

Fernández, Isabel y Hernández, Arturo. "Estudio exploratorio de actitudes en una situación de bilingüismo: el caso mapuche". Revista de Lingüística Teórica y Aplicada 22(1984): 35-52. 
Fishman, Joshua. Language and Ethnicity in Minority Sociolinguistic Perspective. Clevedon: Multilingual Matters, 1989.

Garrett, Paul. Attitudes to Language. Cambridge: Cambridge University Press, 2010.

Gundermann, Hans. "Orgullo cultural y ambivalencia. Actitudes ante la lengua originaria en la sociedad mapuche contemporánea". Revista de Lingüística Teórica y Aplicada 52 (2014): 105-132.

Gundermann, Hans, Canihuan, Jaqueline, Clavería Alejandro y Faúndez, César. Perfil sociolingüístico de comunidades mapuches de la región del Biobío, Araucanía, los Ríos y los Lagos. Informe de investigación. Santiago,Chile: CONADI-UTEM, 2008.

Gundermann, Hans, Canihuan, Jaqueline, Clavería, Alejandro y Faúndez, César. 2011. "El mapuzungun, una lengua en retroceso". Atenea 503 (2011): 111-131.

Henríquez, Marisol. "Estado del Mapudungun en Comunidades Pewenches y Lafkenches de la región del Bio-Bio: El caso de los escolares". Revista de Lingüística Teórica y Aplicada 52 (2014):13-40

Henríquez, M. "Ámbitos de uso del Mapudungun en comunidades Pewenches y Lafkenches de la Región del Bío-Bío".Literatura y Lingüística 31(2015):185204.

Henríquez, Marisol. "Vitalidad de la fonología segmental del chedungun hablado por escolares del Alto Bio-Bio". Alpha 41 (2015): 207-231.

Hernández Campoy, Juan. El fenómeno de las actitudes y su medición en sociolingüística. Murcia: Universidad de Murcia, 2004.

Kroskrity, P. y Field, M. Native American Language Ideologies: Beliefs, Practices, and Struggles in Indian Country. Tucson, University of Arizona Press, 2009.

López Morales, Humberto. Sociolingüística. Madrid: Gredos, 2004.

Lagos, Cristian. "Mapudungun en Santiago de Chile. Vitalidad, lealtad y actitudes lingüísticas". Lenguas Modernas 31 (2006): 97-126.

Moreno Fernández, Francisco. Principios de sociolingüística y sociología del lenguaje. Barcelona: Ariel, 2009.

Ojeda, Patricia, Álvarez, Javier. "Elementos para la construcción social del Chedungun a partir del discurso en torno a la lengua de hablantes bilingües de la VIII región". Boletín de Filología 49 (2014): 161-185.

Olate, Aldo, Henríquez, Marisol. "Actitudes lingüísticas de profesores mapuche de educación básica. Vigencia y enseñanza del mapudungun en el contexto educativo". Literatura y Lingüística 22 (2010): 10-116.

Olate, Aldo, Alonqueo, P. y Caniguan, J. "Interactividad lingüística castellano/mapudungun de una comunidad rural bilingüe". Alpha 37(2013): 265-284.

Olate, Aldo. "Contacto lingüístico mapuzungun/castellano. Aspectos históricos, sociales y lingüísticos. Revisión bibliográfica y propuesta de análisis desde la dimensión morfosintáctica y tipológica”. Onomázein 36(2017): 122-158.

Serrano, María José. Sociolingüística. Barcelona: Ediciones Serbal, 2011.

Silva-Corvalán, Carmen y Arias, Andrés Enrique. Sociolingüística y pragmática del español. Washington: Georgetown University Press, 2017.

Zúñiga, Fernando y Aldo Olate. "El estado de la lengua mapuche, diez años después". El pueblo Mapuche en el siglo XXI. Eds. Isabel Aninat, Verónica Figueroa y Ricardo González. Propuestas para un nuevo entendimiento entre culturas en Chile. Santiago: Centro de Estudios Públicos, 2017. 345-373. 